\title{
Research on Assembly Technology of Active Magnetic Bearing system Based on Graph Theory
}

\author{
ZHANG Li ${ }^{1}$, REN Yafeng ${ }^{2}$, YU Meiyun ${ }^{1}$, LIU Jun ${ }^{1}$, LUO Yanyan ${ }^{1}$ \\ ${ }_{1}^{1}$ AVIC Qingan group co., LTD., 710077, Xian, China \\ ${ }^{2}$ Northwestern Polytechnical University 710072, Xian, China
}

\begin{abstract}
Active magnetic bearing system is a typical geometric constraint system which is combined with each part by certain assembly constraint relations. Graph theory is used to build the assembly model in the progress of assembly design. Trough the graph to express this system, it is not only clear and intuitive, and the algorithm is stable and high efficiency. Modeling active magnetic bearing assembly system is achieved by hierarchical model of data structure,which leads to accelerate the assessment of system configuration program and save a lot of time for designers.
\end{abstract}

\section{Introduction}

Active Magnetic Bearing (AMB) is a typical mechatronic system, which has no contact, wear and lubrication, and easy to controll its dynamic behavior and other series of characteristics[1]. The limitation of traditional mechanical bearing has been breakthrough, it has been widely attracted since it appears. In design of Active Magnetic Bearing system(AMBs), the component and configuration research is very important, and involves the spatial layout and assembly problem. Assembly design of AMBs is defined as dependent on the design method of predefined components or parts. Graph theory[2] are applied to build the assembly model of AMBs, it can quickly achieve the system configuration program. For the first time, Fudos and Hoffmann[3-5] have introduced the concept of cluster into the geometric constraint graph and solved the problem of assembly constraint by geometric constraint graph. N.abe[6] and others have developed a visualization system for assembly analysis and performance evaluation of mechanical system assembly. In the early stage of the design for assembly, this way that is advantageous to analyze the spatial structure and interference will led to accelerate the assessment of system configuration program, which save a lot of time for designers and improve the design efficiency.

\section{Assembly Model of AMBs}

Geometric constraint graph theory is the combination of graph theory and computer graphics. In the field of Graph theory, Geometric Constraint Graph (GCG) is a method that is used to model a geometric constraint system, which can directly manage or express the $2 \mathrm{D}$ or $3 \mathrm{D}$ space of entities and geometric constraints, and has good geometric space expanding and interpretability. In fact, AMBs is a typical geometric constraint system which is combined with each part by a certain assembly constraint relations. Through the graph to express system, it is not only clear and intuitive, and the algorithm is stable and high efficiency. Due to the magnetic bearing system with more parts and complex structures, the constraint relations of assembly model are complex. In terms of expression, maintenance and explanatory, the ideal assembly model has a consistency, which requires the establishment of effective assembly model.

The optimization process of assembly model of AMBs is set up in the process to turn relational model into a hierarchical model. Hierarchical model is consistency with the hierarchical structure of the product assembly in CAD system, which will be beneficial for modeling AMBs by CAD system. There is a kind of effective method to solve the assembly constraints of complex system. when AMBs is abstracted as geometric constraint graph, It is separated into solving the subproblem, at the same time it is suitable for establishing strategy of solving the sub-problem. Eq. (1) is the mathematical expressions for geometric constraint system.

$$
S=(X, C, A)
$$

where, $\mathrm{S}$ is a Active Magnetic Bearing system. $\mathrm{X}$ is a collection of parametric parts. $\mathrm{C}$ is looked as a collection of geometric constraints. $\mathrm{A}$ is parameter values of geometric constraints. The following Eq. (2) is the mathematical expressions for geometric constraint graph.

$$
G C G=(V, E)
$$

GCG is a geometric constraint graph of AMBs. $V$ is the GCG vertex and the same as X. Each vertex is a 
geometric entity. E is the line of GCG, same as C. Each line represents a geometric constraint sets. The assembly model is analyzed by five freedom AMBs, as shown in figure 1.The system is mainly composed of radial magnetic bearing(RMB), thrust magnetic bearing(TMB), sensor, motor, and other key parts[7].

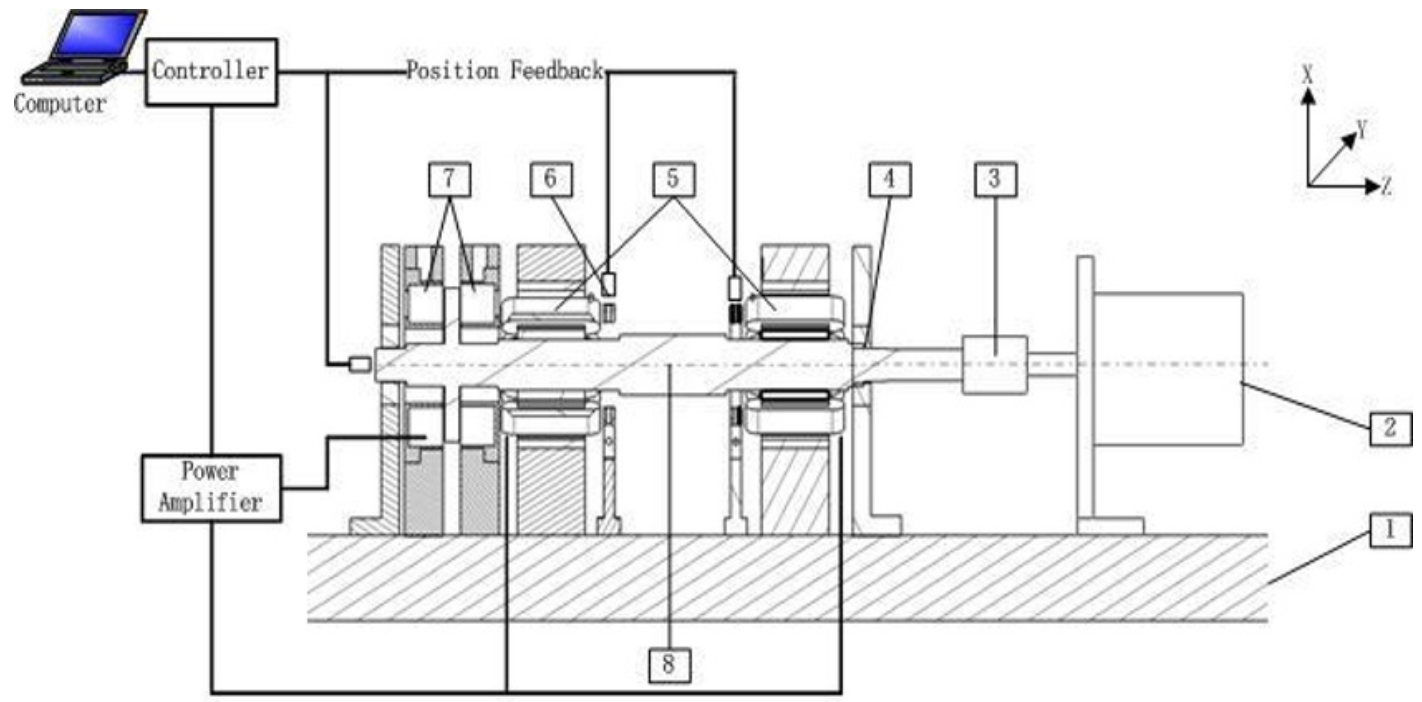

1 Platform 2 Yotor 3 Coupling 4 Touch-down bearing 5 RUB 6 Sensor 7 TMB 8 rotor

Figure 1. Five degrees of freedom AMBs
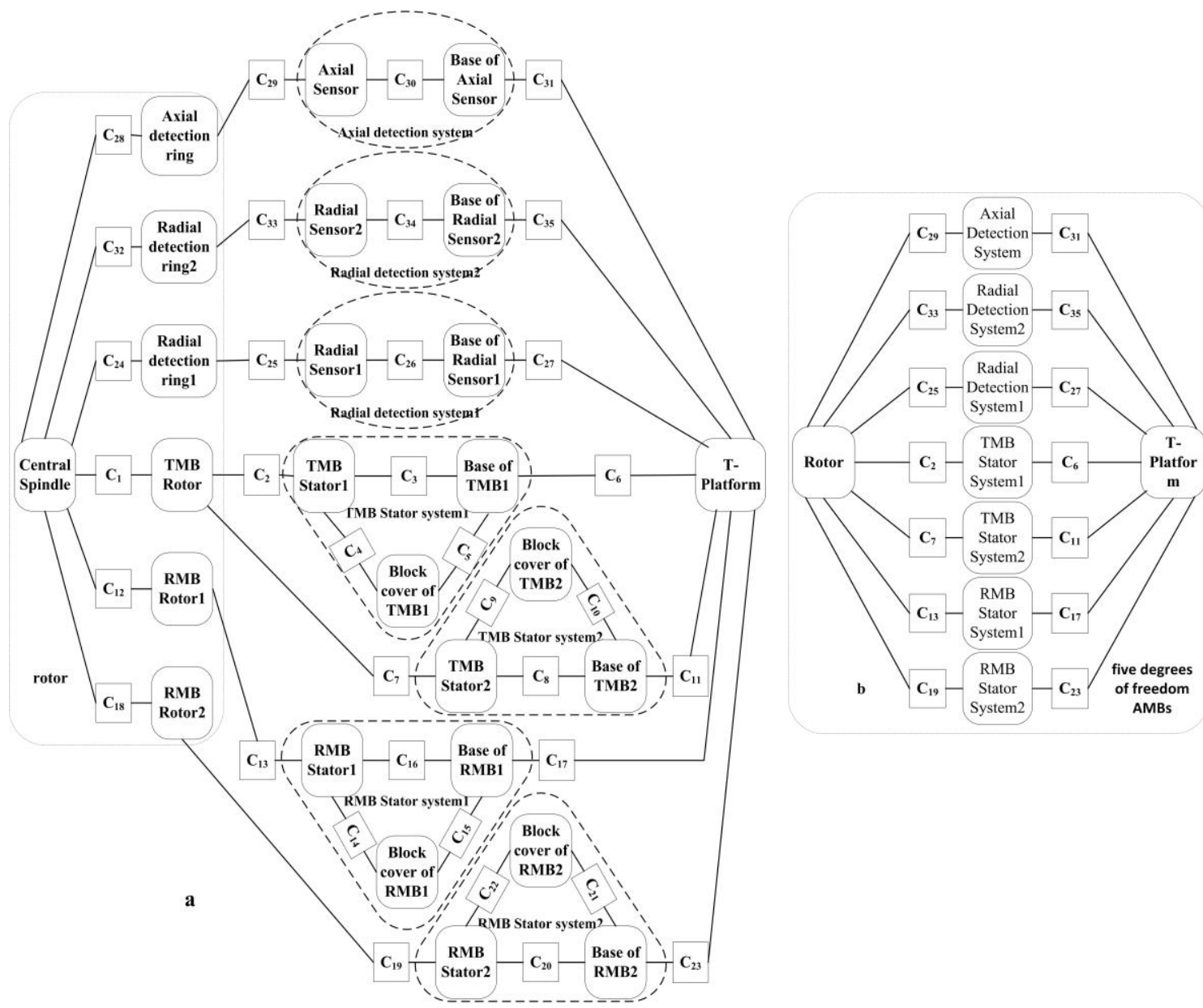

Figure 2. relational model and decomposing process diagram of AMBs. 
Figure 2 is the relational model of five degrees of freedom AMBs. From figure $2 \mathrm{a}$ to figure $2 \mathrm{c}$ reflects the process of polymerization contraction between the assembly model. There are two kinds of nodes in the graph. Oval node represents component that contains modeling parameters, such as the stator or rotor, etc. Square node is the assembly constraint relations between components, for example $\mathrm{C} 1$ is an assembly constraint set between the central spindle and TMB rotor. The circle part that is dashed reflects a condensed and aggregated node called new node. It is the basic component of system structure. The component is an assembly unit to be achieved a certain function, which is a part or multiple parts.

In a new node, the distribution of constraints is changed and it results in the system assembly size to be reduced. It is called the decomposition process of geometric constraint system that separating from external constraints into the internal constraints. Specific speaking,
$\mathrm{C} 1, \mathrm{C} 12, \mathrm{C} 18, \mathrm{C} 24, \mathrm{C} 28$ and $\mathrm{C} 32$ are converted into the rotor,s internal constraints. $\mathrm{C} 3, \mathrm{C} 4$ and $\mathrm{C} 5$ are converted into the internal constraints of TMB stator1. $\mathrm{C} 8, \mathrm{C} 9$ and $\mathrm{C} 10$ are converted into the internal constraints of TMB stator2. $\mathrm{C} 14, \mathrm{C} 15$ and $\mathrm{C} 16$ are converted into the internal constraints of RMB stator1.New node is replaced to form a new geometric constraint graph, it is a shown in the figure $2 \mathrm{~b}$. In the process of the second decomposition, the new graph can be integrated as a node, as shown in figure $2 \mathrm{c}$.

From the decomposition process of relational model, hierarchical model of AMBs has be concluded. More importantly, the hierarchical model is used to model the assembly in CAD system, as shown in figure 3. This assembly model is divided into three layers. The first layer is the top level, which is as the general assembly. The second layer is seen as a sub- assembly. The last layer is the parts, which is the AMBs foundation.

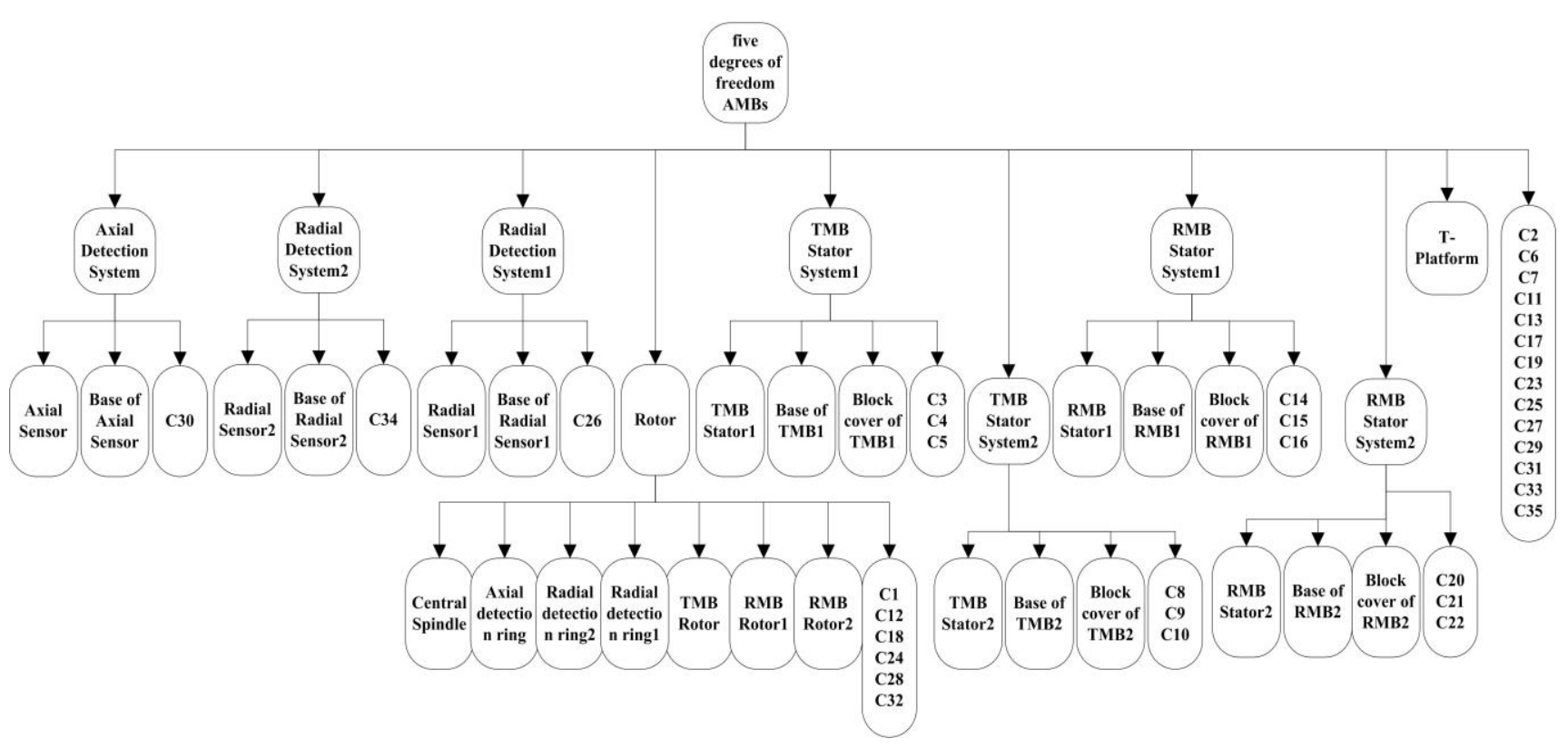

Figure 3. hierarchical model of AMBs.

\section{Data Structure of AMBs}

In the assembly model of AMBs, the node is relatively independent assembly unit, such as sub-assembly or component. The node is regarded as an object to define properties. The result is data structure of AMBs that includes assembly constraint relations, hierarchical structure and material properties. The following is a data structure.

a. Assembly:

Name: the name of AMBs

ID: the unique identification of AMBs

Context: it contains all of Subassembly ID

Pointer: it points to Subassembly

b. Subassembly:

Name: the name of Subassembly

ID: the unique identification of Subassembly
Context: it contains all of Component ID

Pointer: it points to Component

c. Component:

Name: the name of Component

ID: the unique identification of Component

Context: it contains all of Part ID

Pointer: it points to Part

d. Part:

Name: the name of Part

ID: the unique identification of Part

Parameter: the parameters of Part

Material: the material property of Part

e. Constraint:

Name: the name of Constraint

Type: the type of Constraint

Assembly is the top node that reflects the general assembly of AMBs, which includes Name, ID, Context 
and Pointer. The Pointer describes the direction of the next layer between assembly Constraints. The Subassembly is the second decomposition node. In fact, when the second aggregation is divided, designers interfere the subassembly in an interactive way, but to be following a certain principle. The component is a basic assembly unit of AMBs. In the interactive assembly, the component from a component library is used to mix design. The Part is the bottom of the basic component. The Constraint corresponds to the constraint in CAD system. it is advantageous to the depth of the secondary development.

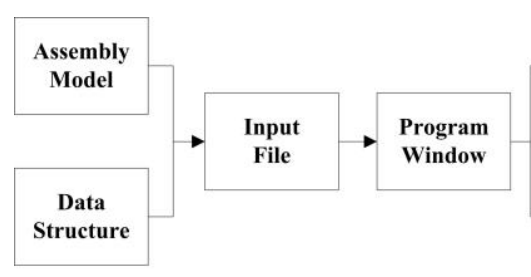

\section{Modeling of AMBs}

Based on the assembly model and data structure, the input files for AMBs are designed .The corresponding windows are customized to realize the secondary development of CATIA by VB language. Program drives the input file to obtain the assembly information that are modeling parameters, constraints and material, etc. Basic component library and CATIA API interface are used to design a $3 \mathrm{D}$ model about AMBs. This $3 \mathrm{D}$ model is a preassembly model to be adjusted and modified. Figure 4 is the assembly modeling flowchart of AMBs.

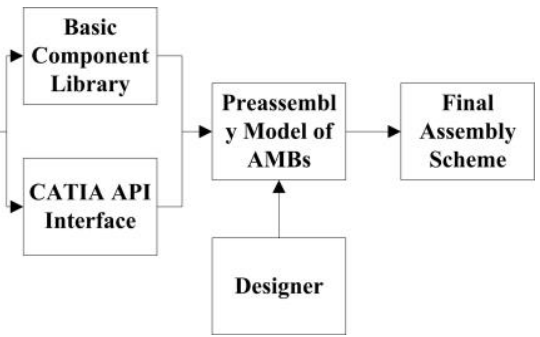

Figure 4. assembly modeling flowchart of AMBs.

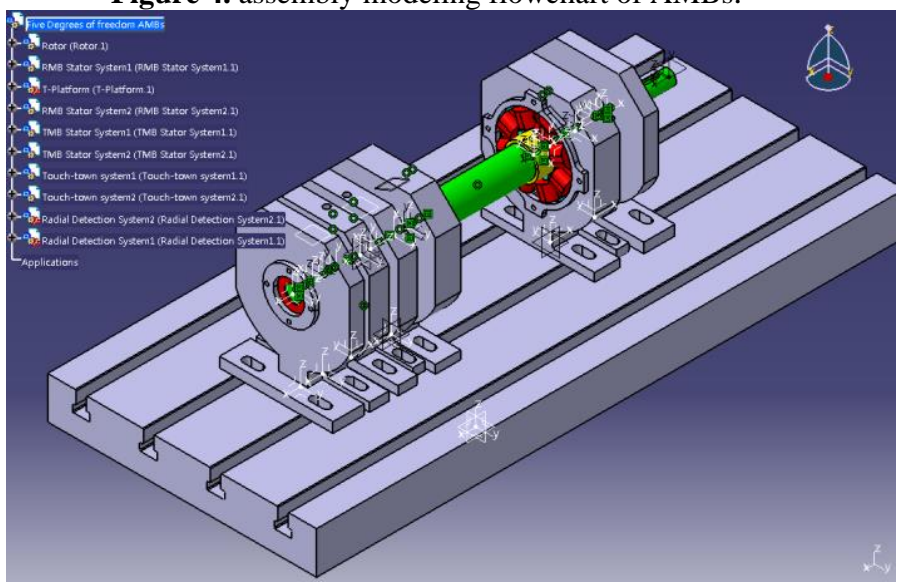

Figure 5. 3D model of AMBs.

According to the assembly modeling flowchart of AMBs, The top-down assembly design way is adopted to assembly 3D model in CATIA, as shown in figure 5.

\section{Conclusion}

Graph theory are applied to build the assembly model of AMBs, it can quickly achieve the system configuration program. In an early stage of the design for assembly,

\section{References}

1. G.Schweitzer, H.Maslen. Magnetic bearing-Theory, design, and application to rational machinery [M], 2009

2. Koh Khee Meng. Introduction to Graph Theory [M], 2007

3. Fudos I, Hoffmann C M. A graph-constructive approach to solving systems of geometric constraints[J]. ACM Transaction on Graph, 1997, 16(2): 179 216

4. Bouma W, Fudos I, Hoffmann C M, et al. Geometric constraint solver[J]. Computer Aided Design, 1995,27(6):487-501 this way that is advantageous to analyze the spatial structure and interference will led to accelerate the assessment of system configuration program, which save a lot of time for designers and improve the design efficiency.

5. Fudos I, Hoffmann C M. Correctness proof of a geometric constraint solver. Technical Report[J]. Dept of Computer Science , Purdue University, USA, 1993

6. N.Abe,et al. Verification of assembly ability of mechanical parts and visualization of machinery of assembly in virtual space $[\mathrm{J}]$. Proceedings of International Conference on Virtual Reality ,1997

7. Andrea Tonoli, Angelo Bonfitto, Mario Silvagni and Lester D. Suarez. Rotors on Active Magnetic Bearings[J]: Modeling and Control Techniques, 2012. 